\title{
Successful Treatment of Primary Aldosteronism With Partial Adrenalectomy, Facilitated By The Use of ${ }^{11} \mathrm{C}$-Metomidate PET/CT
}

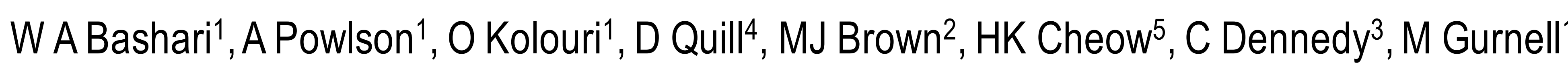

${ }^{1}$ Institute of Metabolic Science \& ${ }^{2}$ Department of Clinical Pharmacology, University of Cambridge \& Addenbrooke's Hospital, Cambridge, UK

${ }^{3}$ Department of Endocrinology \& 4 Department of Surgery, University Hospital Galway, Galway, Ireland ${ }^{5}$ Department of Radiology, Addenbrooke's Hospital, Cambridge, UK

\section{Background}

Primary aldosteronism (PA) is estimated to be responsible for $5-10 \%$ of all cases of hypertension (HTN) ${ }^{1}$. The current gold standard test for determining lateralisation in PA is adrenal vein sampling (AVS). ${ }^{11} \mathrm{C}$-Metomidate PET/CT (MTO-PET) has recently emerged as a potential non-invasive alternative to AVS ${ }^{2}$. As ${ }^{11} \mathrm{C}$-Metomidate is concentrated within 'hyper-functioning' nodules, MTO-PET potentially not only identifies the side, but the exact site of aldosterone hypersecretion, thus raising the possibility of more targeted surgical intervention.

\section{Case Report}

A 45-year-old man was noted to have HTN and hypokalaemia following a myocardial infarction. He required four anti-hypertensive agents to achieve BP control. His plasma aldosterone was elevated with a suppressed plasma renin (off interfering medications), and aldosterone did not adequately decrease following saline suppression, confirming the diagnosis of PA. Adrenal CT and MRI did not convincingly demonstrate a lesion. He underwent AVS, but the result was inconclusive (right adrenal vein not cannulated). MTO-PET revealed focally increased tracer uptake in a sub-centimetre nodule in the left adrenal gland.

\section{Investigations}

\section{ARR screening:}

\begin{tabular}{|c|c|c|}
\hline Renin & $<2$ & $\mathrm{mU} / \mathrm{L}$ \\
\hline Aldosterone & 932 & $\mathrm{pmol} / \mathrm{L}$ \\
\hline
\end{tabular}

Saline infusion test:

\begin{tabular}{|c|c|c|c|}
\hline Time & Aldosterone & Renin & Cortisol \\
\hline Oh & 934 & $<0.2$ & 586 \\
\hline$+4 \mathrm{~h}$ & 584 & $<0.2$ & 213 \\
\hline
\end{tabular}

\section{Adrenal Vein Sampling:}

\begin{tabular}{|c|c|c|c|}
\hline Site & Cortisol & Aldosterone & Aldo:Cort \\
\hline Periphery & 522 & 1028 & 1.97 \\
\hline Left Adrenal & 816 & 37763 & 46.27 \\
\hline Right Adrenal & 487 & 969 & 1.99 \\
\hline
\end{tabular}

\section{CT Adrenal:}
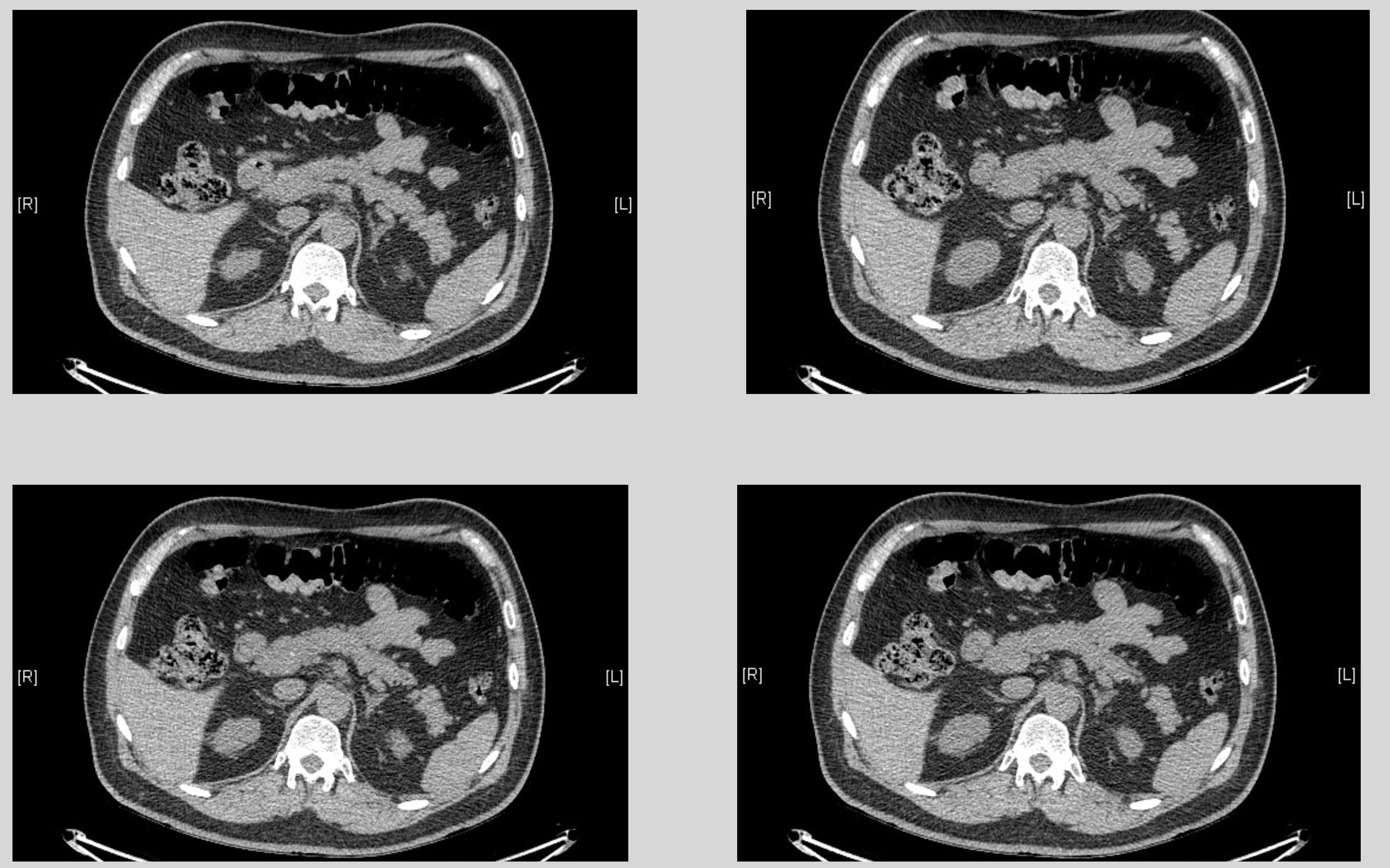

\section{${ }^{11} \mathrm{C}$-Metomidate PET/CT:}

Axial

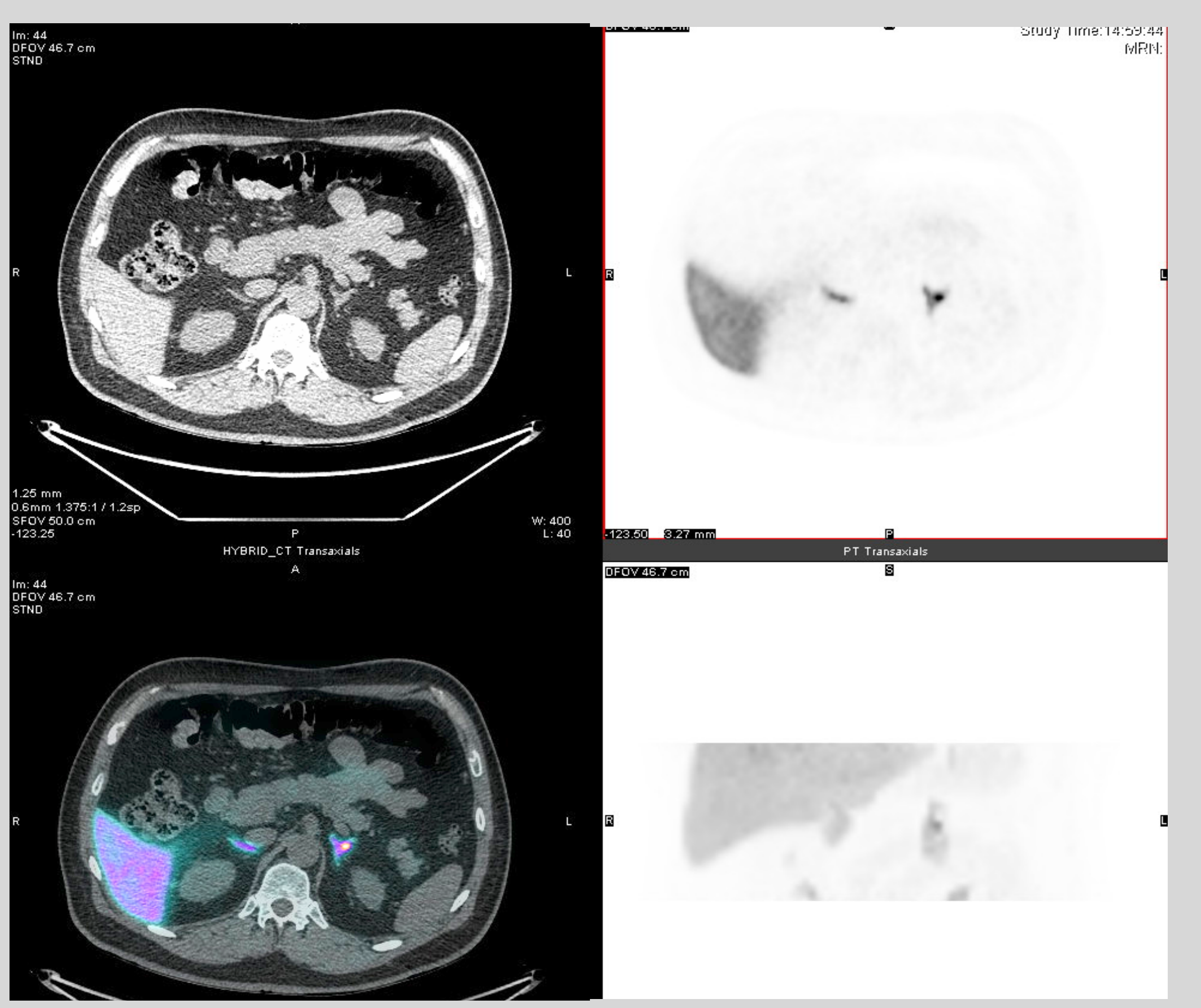

Coronal

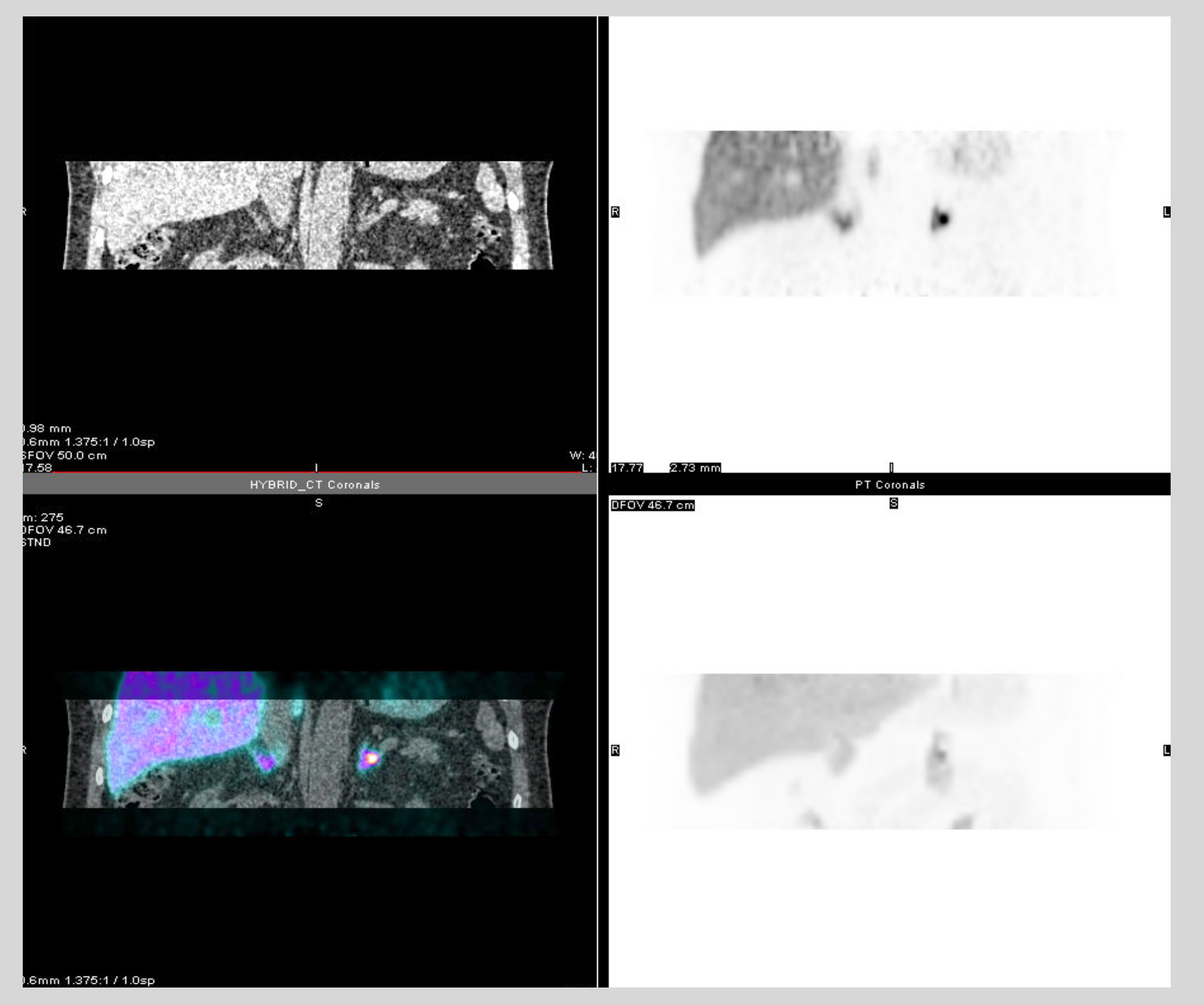

Sagittal

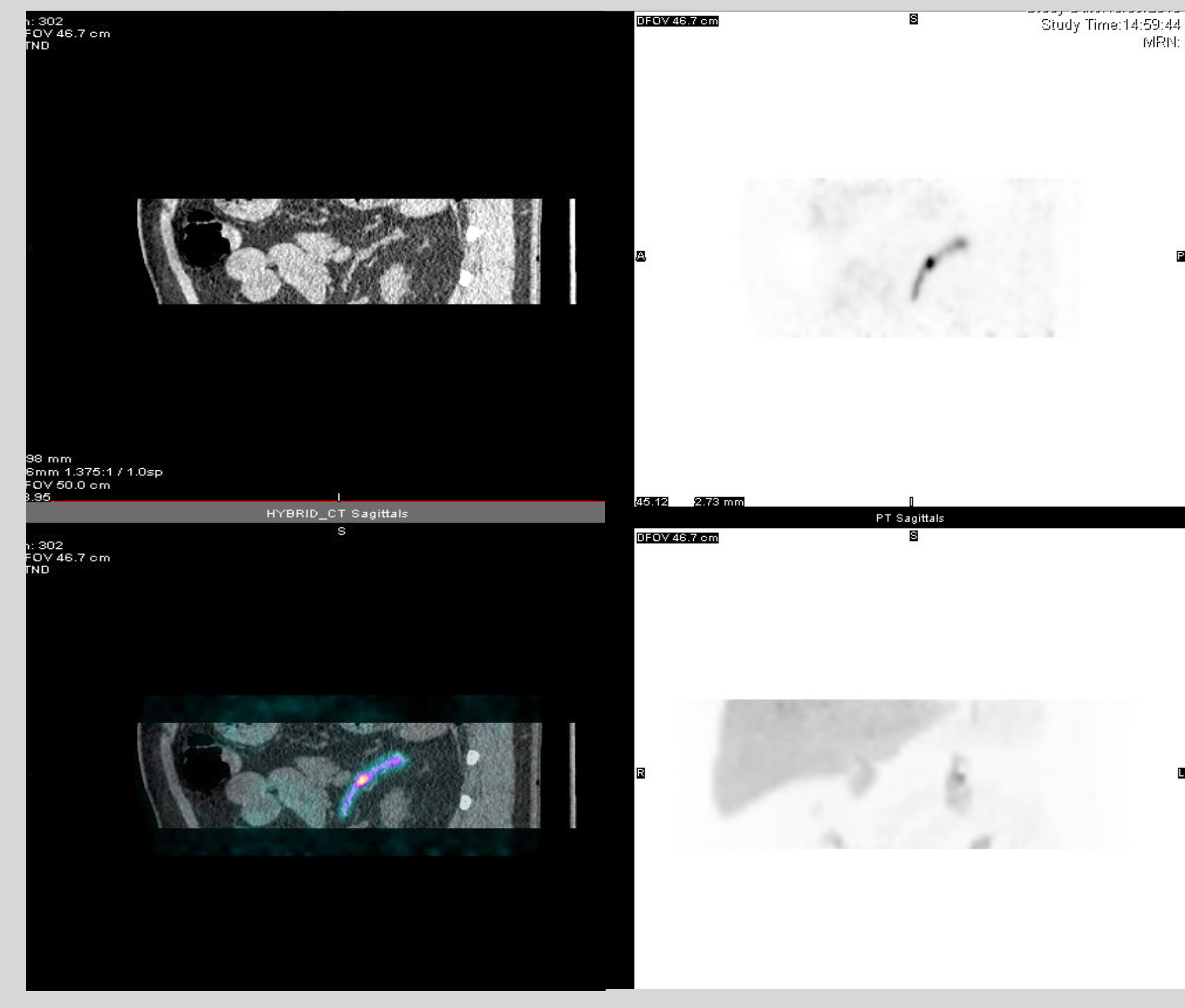

\section{Treatment and outcome}

The patient underwent a posterior retroperitoneoscopic procedure, during which the nodule and lateral limb of the left adrenal were selectively removed, leaving the rest of the gland in situ. Histology confirmed the presence of a small Conn's adenoma. The patient is normotensive post-surgery (BP 110/74 $\mathrm{mmHg}$ ), on no antihypertensive medications, with normal biochemistry.

\begin{tabular}{|c|c|c|c|c|}
\hline & Pre-op & Day 2 & Month 3 & \\
\hline Renin & $<2$ & 63.8 & 30.9 & $\mathrm{mU} / \mathrm{L}$ \\
\hline Aldosterone & 932 & $<102$ & 352 & $\mathrm{pmol} / \mathrm{L}$ \\
\hline
\end{tabular}

\section{Conclusion}

This case highlights the ability of MTO-PET to not only lateralise, but actually localise the site of aldosterone hypersecretion, and thereby guide selective removal of a Conn's adenoma with sparing of the adjacent normal adrenal gland. 